\title{
A versatile DNA-supramolecule logic platform for multifunctional information processing
}

\author{
Chunrong Yang ${ }^{1}$, Lingbo Song ${ }^{2}$, Jianchi Chen ${ }^{1}$, Dan Huang ${ }^{1}$, Junling Deng ${ }^{1}$, Yuanyuan Du ${ }^{3}$ Dehong Yang ${ }^{4}$, \\ Shu Yang ${ }^{2}$, Qianfan Yang ${ }^{1}$ and Yalin Tang ${ }^{5}$
}

\begin{abstract}
DNA-based logic units are rapidly being developed in molecular computation. However, because DNA cannot produce detectable signals, suitable signal reporters must be carefully selected, which is challenging, especially in advanced, multifunctional devices. By introducing a supramolecular reporter MTC [3,3'-di(3-sulfopropyl)-4,5,4',5'dibenzo-9-methyl-thiacarbo-cyanine triethylammonium salt], we developed a simplified DNA-supramolecule platform. Owing to the multiple assembly states of MTC, the platform contains only one reporter block but can provide multiple parallel outputs and easily implement several types of information processing functions, including data filtration (binary and ternary INHIBIT gates), selection (multiplexer and demultiplexer) and verification (parity checker and comparator). In addition to combinational circuits, a fundamental sequential logic circuit, counter, has also been fabricated at the molecular level. With the advantages of high reconfigurability, flexibility and enormous parallelism, this DNA-supramolecule prototype may have a promising future in the field of molecular computing and multiplex chemical analysis.
\end{abstract}

\section{Introduction}

Molecular computation has attracted intense attention in widespread. scientific studies over the past few dec$\operatorname{ades}^{1-3}$. Unlike traditional digital circuitry whose logic units are physically combined, molecular logic units are functional-combined by associating chemical or biochemical molecules ${ }^{4,5}$. In molecular logic devices, DNA is endowed with unique advantages due to its strict base complementarity and controllable structural transitions. Hitherto, a series of advanced logic circuits, such as multiplexer/demultiplexer ${ }^{6}$, encoder/decoder ${ }^{7,8}$ and parity checker ${ }^{9,10}$, have been constructed via DNA-based systems. These works not only promote the development

Correspondence: Shu Yang (yangshu1106@scu.edu.cn) or Qianfan Yang (yangqf@scu.edu.cn)

'College of Chemistry, Sichuan University, Chengdu 610064, China

${ }^{2}$ Key Laboratory of Drug Targeting and Drug Delivery Systems, West China School of Pharmacy, Sichuan University, Chengdu 610041, China

Full list of author information is available at the end of the article. of molecular computation but also show great application potential for biomedical imaging ${ }^{11}$, data storage ${ }^{12}$, medical diagnostics ${ }^{13}$, and biochemical analysis ${ }^{14}$.

Although great advancements have been achieved in DNA computation, the inherent drawback that DNA cannot generate detectable signals still exists. Therefore, the development of DNA-based circuits relies heavily on the design of ingenious signal reporters. Reported DNAbased logic systems mostly contain two independent components: (1) oligonucleotides in charge of logical function and (2) extrinsic reporters responsible for monitoring the "state" of the logic devices. This strategy works very well in simple logic circuits, such as basic Boolean logic gates and some single-output units ${ }^{9,15}$. However, for advanced, multi-output logic circuits, selecting suitable reporters with distinguishable signals is still a challenge. For example, the DNA-based platform designed by Wang's group ${ }^{8}$ employed four different fluorescent reporters with partially overlapping signals to

\section{(c) The Author(s) 2018}

(c) (i) Open Access This article is licensed under a Creative Commons Attribution 4.0 International License, which permits use, sharing, adaptation, distribution and reproduction cc) in any medium or format, as long as you give appropriate credit to the original author(s) and the source, provide a link to the Creative Commons license, and indicate if changes were made. The images or other third party material in this article are included in the article's Creative Commons license, unless indicated otherwise in a credit line to the material. If material is not included in the article's Creative Commons license and your intended use is not permitted by statutory regulation or exceeds the permitted use, you will need to obtain permission directly from the copyright holder. To view a copy of this license, visit http://creativecommons.org/licenses/by/4.0/. 
construct a 2-to-4 decoder. Obviously, the higher the complexity of the logic functions, the more difficult it is to find suitable reporters. To simplify the design of advanced logic circuits, we introduce a supramolecular reporter, which involves only one molecular block but is capable of displaying multiple assembly states ${ }^{16,17}$ and, consequently, represents multiple output channels.

Cyanine dye, a common fluorochrome, has been applied in various devices, such as biosensors ${ }^{18}$, components for nonlinear optics ${ }^{19}$, and data storage devices ${ }^{20}$, owing to its unique photophysical and photochemical properties. Cyanines can assemble into various aggregates ${ }^{21}$, and each aggregate presents distinguishable spectral features, implying that they have the potential to simultaneously provide multiple independent output channels. In our previous work, we succeeded in constructing a 2-to-4 decoder based on a cyanine supramolecular system ${ }^{22}$. Another advantage of cyanines is that their assembly behavior can be regulated by various stimuli, including $\mathrm{pH}$, metal ions and biomacromolecules, especially specific DNA structures ${ }^{23-28}$, and the assembly conversions could be reversed by eliminating the stimuli. Herein, we developed a multifunctional logic platform based on DNA D1 (5'-GGTGGTGGTGGT-3'), D2 (5'-CCACCACCACCACAACCACCACCACCAAA-3'), and cyanine dye $M T C$ [3,3'-di(3-sulfopropyl)-4,5,4',5'-dibenzo-9-methyl-thiacarbocyanine triethylammonium salt] (Fig. 1). By introducing stimuli that can regulate both the secondary structures of $D 1 / D 2$ and the assembly states of $M T C$, the platform implemented several types of information processing functions, including data filtration, selection, verification, and accumulation.

\section{Materials and methods}

\section{Chemicals and reagents}

DNA oligonucleotides D1 (5'-GGTGGTGGTGGT-3') and D2 (5'-CCACCACCACCACAACCACCACCAC CAAA-3') were purchased from Sangon Biotechnology Co. Ltd. (Shanghai, China). The cyanine dye MTC was synthesized according to the methods of $\mathrm{Hamer}^{29}$ and Ficken $^{30}$, and the purity was determined by MS, elemental analysis, NMR and absorption spectroscopy (shown in Figs. S1-S4 and Tables S1-S3). Lead chloride $\left(\mathrm{PbCl}_{2}\right)$, 18crown-6-ether $(\mathrm{CE})$ and potassium nitrate $\left(\mathrm{KNO}_{3}\right)$ were purchased from Aldrich Ltd. (Shanghai, China). Silver nitrate $\left(\mathrm{AgNO}_{3}\right)$ and L-cysteine (Cys) were obtained from Sigma-Aldrich. All the chemicals are analytical reagent grade and used as received without further purification. Ultrapure water prepared by an ULUPURE (Chengdu,

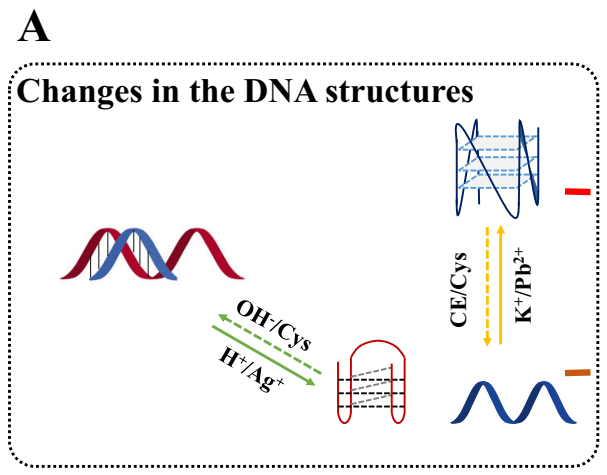

B

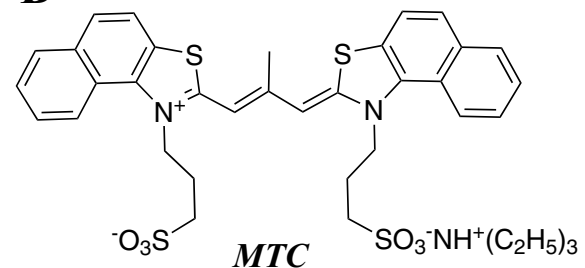

MTC
J-aggregates

Monomer
H-aggregates

Dimer

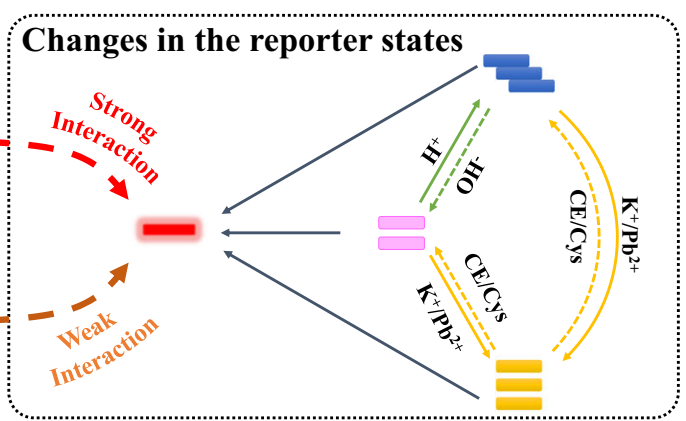

C
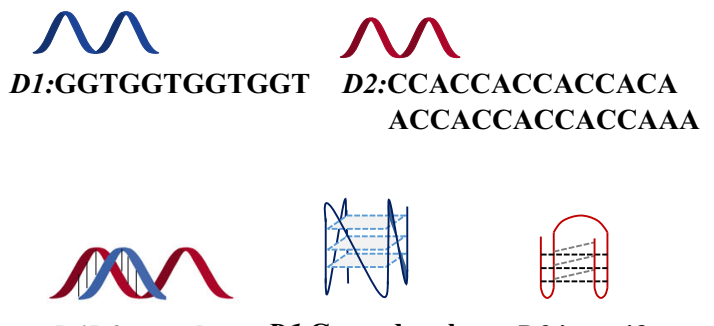

D1D2 complex D1 G-quadruplex D2 i-motif

Fig. 1 Scheme of the DNA-supramolecule logic platform. a The changes in the structures of D1/D2 and the assembly states of MTC triggered by various inputs. $\mathbf{b}$ The molecular structure and assembly states of MTC. $\mathbf{c}$ The DNA motifs of D1 and D2 
China) ultrapure water system and was used throughout the experiments.

The stock solution of $M T C$ was prepared by dissolving its powder in methanol and was stored in darkness. The DNA stock solutions were prepared by dissolving certain amounts of oligonucleotides in $10 \mathrm{mM}$ Tris- $\mathrm{HCl}$ buffer solution. The $D 1 D 2$ duplex was prepared by mixing $D 1$ and $D 2$ in equimolar amounts and annealing the mixture in a thermocycler (first heated at $90^{\circ} \mathrm{C}$ for $5 \mathrm{~min}$ and then cooled slowly to room temperature). The DNA concentrations were determined by measuring the absorbance at $260 \mathrm{~nm}$.

The fluorescence spectra were collected with a F-4600 spectrophotometer (HITACHI, Japan) in a $10 \mathrm{~mm}$ quartz cell. Xenon arc lamp was used as the excitation light source. Both the excitation and emission slits were $5 \mathrm{~nm}$, the PMT voltage was $400 \mathrm{~V}$, and the excitation wavelength was $530 \mathrm{~nm}$. The absorption spectra were acquired with an EVOLUTION 201 spectrophotometer (Thermo SCIENTIFIC, USA) at room temperature in a $10 \mathrm{~mm}$ quartz cell.

\section{Operations of the logic circuits}

To better demonstrate the logic functions of the platform, the optimized input conditions were selected for each circuit. All samples were incubated in the dark at room temperature for $20 \mathrm{~min}$ before the measurement.

For the binary INHIBIT gate, $4 \mu \mathrm{M} D 1$ and $4 \mu \mathrm{M} M T C$ in $10 \mathrm{mM}$ Tris- $\mathrm{HCl}$ buffer solution ( $\mathrm{pH}$ 7.0) were used as the initial state, and $10 \mu \mathrm{M} \mathrm{PbCl}$ and $4 \mu \mathrm{M} \mathrm{D} 2$ were employed as the inputs. With respect to the ternary INHIBIT gate, $4 \mu \mathrm{M} M T C$ in $10 \mathrm{mM}$ Tris-Ac buffer solution ( $\mathrm{pH}$ 7.4) was used as the initial state, and $1 \mu \mathrm{M}$ $D 1,1 \mu \mathrm{M} \mathrm{D1}$ with $0.15 \mu \mathrm{M} \mathrm{PbCl}_{2}, 1 \mu \mathrm{M} \mathrm{D} 2$, and $1 \mu \mathrm{M} \mathrm{D} 2$ with $3 \mu \mathrm{M} \mathrm{AgNO} 3$ were employed as the inputs.

For the 2:1 MUX, $4 \mu \mathrm{M} \mathrm{D1}$ and $4 \mu \mathrm{M} \mathrm{MTC}$ in $10 \mathrm{mM}$ Tris- $\mathrm{HCl}$ buffer solution ( $\mathrm{pH}$ 7.0) were used as the initial state; $10 \mu \mathrm{M} \mathrm{PbCl} 2$ and $10 \mathrm{mM}$ Tris- $\mathrm{HCl}$ buffer solution (pH 4.0) were employed as the data inputs; and $4 \mu \mathrm{M} \mathrm{D2}$ with $30 \mathrm{mM} \mathrm{KCl}$ was employed as the address input.

For the DEMUX and parity checker, $6 \mu \mathrm{M} \mathrm{MTC}$ in 10 $\mathrm{mM}$ Tris- $\mathrm{HCl}$ buffer solution ( $\mathrm{pH}$ 8.5) was used as the initial state; $60 \mathrm{mM} \mathrm{KCl}$ was employed as the data input; and $50 \mathrm{mM} \mathrm{CE}$ and $6 \mu \mathrm{M} \mathrm{D1}$ were used as the address inputs in the 1:3 DEMUX. $10 \mathrm{mM}$ Tris- $\mathrm{HCl}$ buffer solution (pH 4.0), $60 \mathrm{mM} \mathrm{KCl}, 10 \mu \mathrm{M} \mathrm{PbCl}{ }_{2}$ and $D 1$ were employed as the four binary bits of the examined numbers in the parity checker.

For the comparator, $6 \mu \mathrm{M} \mathrm{MTC}$ and $6 \mu \mathrm{M} \mathrm{D1D2}$ in 10 $\mathrm{mM}$ Tris- $\mathrm{HCl}(\mathrm{pH} 8.5)$ were used as the initial state, and $30 \mathrm{mM} \mathrm{KCl}$ and $10 \mathrm{mM}$ Tris- $\mathrm{HCl}$ buffer solution (pH 4.0) were employed as the inputs.

For the counter, $5 \mu \mathrm{M} \mathrm{MTC}$ in $10 \mathrm{mM}$ Tris- $\mathrm{HCl}$ buffer solution ( $\mathrm{pH} 8.5$ ) was used as the initial state; $40 \mathrm{mM} \mathrm{KCl}$ and $70 \mathrm{mM} \mathrm{CE}$ were selected as the cumulating and descending pulses, respectively.

\section{Operations of the logic circuits in human serum}

The human serum samples were supplied by Solarbio (China). The serum was centrifuged at 10,000 rpm for 3 min to remove precipitates, and the supernatants were diluted ten times using Tris- $\mathrm{HCl}(10 \mathrm{mM}, \mathrm{pH}$ 7.0) buffer before the logic operations. For the logic operations, all the samples contained 1000-fold diluted serum, and the input stimuli were consistent with the above description.

\section{Results and discussion}

As a typical cyanine, MTC presents several assembly behaviors under different conditions. In alkaline buffer solutions (such as $\mathrm{pH} 8.5,10 \mathrm{mM}$ Tris- $\mathrm{HCl}$ ), it has a dominating absorption peak at $528 \mathrm{~nm}$ assigned to dimer (termed the D-band), while in acidic conditions (such as $\mathrm{pH} 4,10 \mathrm{mM}$ Tris- $\mathrm{HCl}$ ) it self-assembles into J-aggregates (typical absorption peak at approximately $655 \mathrm{~nm}$, termed the J-band) ${ }^{31}$, as shown in Supplementary Figs. S5 and S6. Some cations can also facilitate the self-assembly of $M T C$. For example, $M T C$ can assemble into either J- or $\mathrm{H}$ aggregates (absorption peak in the range of $440-480 \mathrm{~nm}$, termed the H-band) in the presence of potassium (Supplementary Fig. S7). In addition, we previously reported that certain DNA G-quadruplex structures can specifically disassemble MTC aggregates into monomers, accompanied by a dramatic fluorescence enhancement (maximum emission wavelength at $600 \mathrm{~nm}$, termed the $\mathrm{FI})^{32,33}$. The unique assembly behavior of $M T C$ can provide at least four distinguishable output signals (D-band, J-band, $\mathrm{H}$-band, and FI), which makes it convenient for the construction of multi-output logic circuits.

In this paper, we defined the threshold of the output signals (normalized MTC J-band, $\mathrm{H}$-band or FI) at 0.3 in all the binary logic devices. Any signal greater than the threshold was defined as "1", and those less than the threshold as "0". Similarly, the thresholds in the ternary gate were defined at 0.3 and 1.5. It is worth mentioning that all the inputs in this work can be eliminated by adjusting the complexing agents, so all the logic operations are reversible. For example, $\mathrm{Pb}^{2+}$ and $\mathrm{Ag}^{+}$can be complexed by cysteine (Cys), $\mathrm{K}^{+}$can be complexed by 18 crown-6 (CE), and the G-quadruplexes can be unwound into a stable complex (Supplementary Figs. S8 and S9).

\section{Data filtration: the binary and ternary INHIBIT gates}

The INHIBIT gate (Fig. 2a) is one of the most widely used Boolean logic gates. A binary INHIBIT gate can only output the high level by inputting special one of the two inputs individually. This filtering function makes it to be applied widely in biochemical detection ${ }^{13}$. As mentioned above, MTC can recognize specific G-quadruplex 
structures against single-/double-stranded oligonucleotides. The G-rich oligonucleotide $D 1$ in a random single strand (under metal ion-free conditions) can induce only weak fluorescence signal of $M T C$, while its parallel Gquadruplex formation (induced by metal ions, such as $\mathrm{K}^{+}$ or $\mathrm{Pb}^{2+}$ ) can cause a strong fluorescence enhancement. In addition, the D1D2 complex can hardly induce fluorescence (Supplementary Fig. S8). By utilizing distinctive fluorescence patterns, both binary and ternary logic gates were constructed.

To construct a binary INHIBIT gate, we chose MTC and $D 1$ as the initial state and $\mathrm{Pb}^{2+}$ and $D 2$ as the input

A

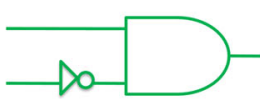

B

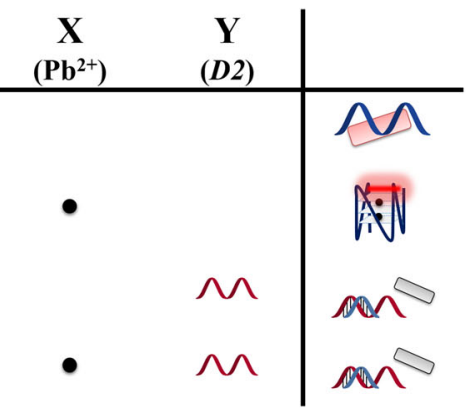

C

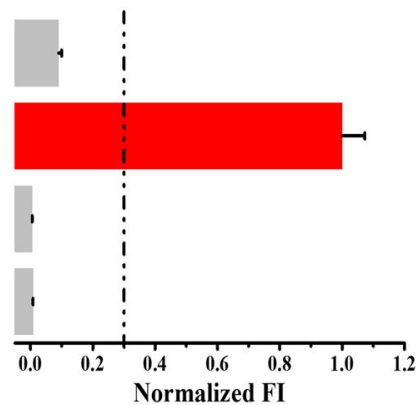

D

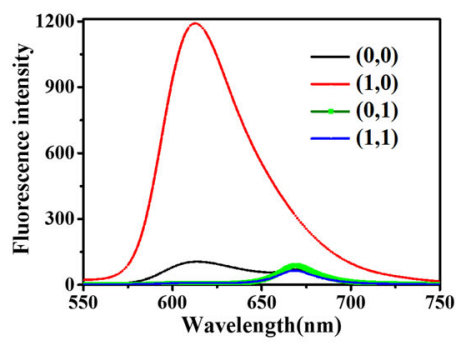

E
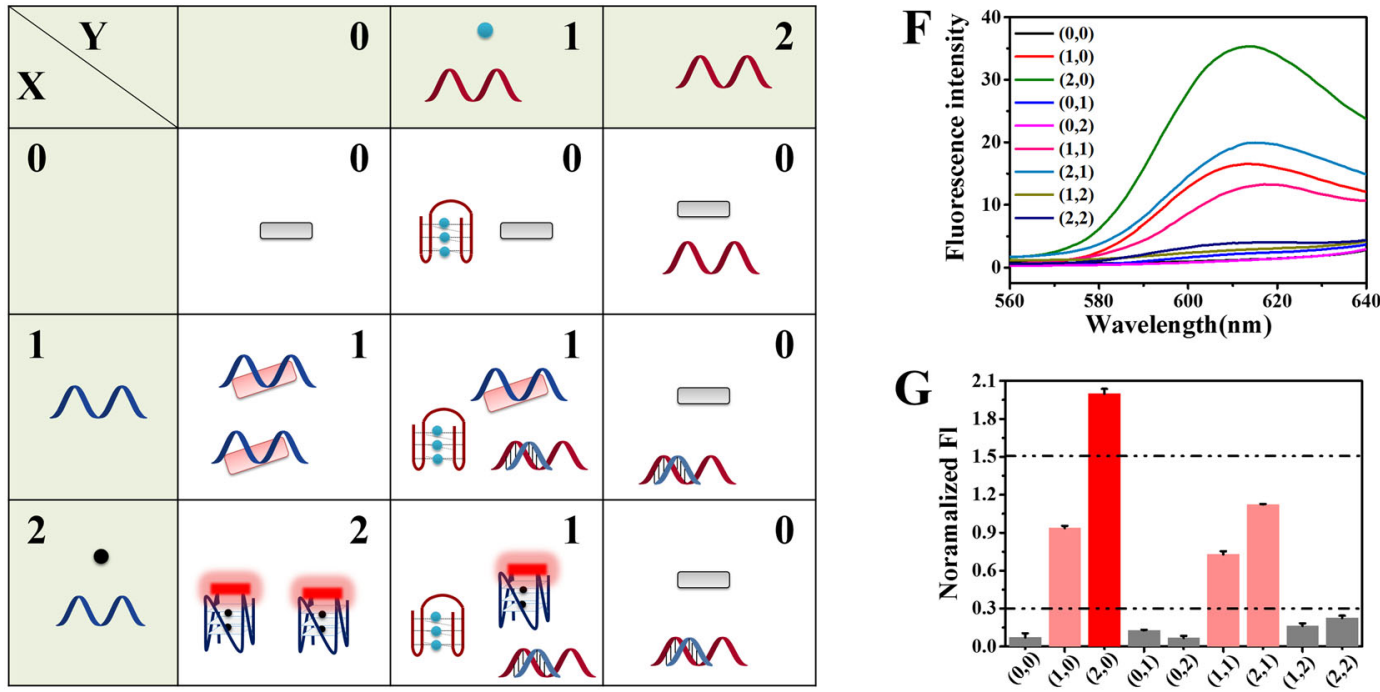

G

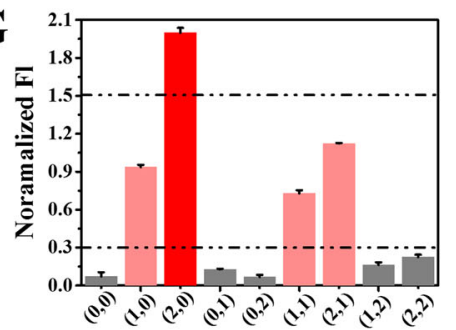

Fig. 2 Schematic illustration of the INHIBIT gates. a Diagram of the electronic INHIBIT logic circuit. $\mathbf{b}$ The schematic truth table of the binary INHIBIT gate. The fluorescence spectra of MTC with different input conditions (c) and the normalized fluorescence intensity (FI) of MTC monomers as the output (d) in the binary INHIBIT gate. e The schematic truth table of the ternary INHIBIT gate. The fluorescence spectra of MTC with different input conditions (f) and the normalized MTC FI as the output $(\mathbf{g})$ in the ternary INHIBIT gate 
stimuli (Fig. 2b). $\mathrm{Pb}^{2+}$ can facilitate the formation of the D1 G-quadruplex, while D2 has the opposite effect. As shown in Fig. 2d, in the initial state, MTC shows a weak fluorescence signal with single-stranded $D 1$, while it emits a strong signal with $\mathrm{PbCl}_{2}$ input. The addition of D2 complements $D 1$ to $D 1 D 2$ complex, which is stable and cannot be unwound by $\mathrm{Pb}^{2+}$, and reduces the signal to near zero. The normalized MTC FI values are plotted as a bar chart in Fig. 2c, and the result is accordance with the proper execution of the INHIBIT gate.

In binary logic systems, each bit is coded by " 0 " or " 1 ", corresponding to a low and high signal, respectively. However, due to the complexity of information processing, sometimes it is difficult to identify certain and accurate states based on this low/high system. In contrast, multi-valued logic systems, which involve a switch between multiple states, show an overwhelming advantage in dealing with uncertain information. Ternary logic gates, as a typical representation of multi-valued logic systems, have three different output levels: low, medium and high, which correspond to logic values of " 0 ", " 1 ", and "2", respectively ${ }^{34}$. With respect to $M T C$, its fluorescence signals can be induced to three different levels by $D 1$ with certain structures, i.e., (1) a strong signal induced by the G-quadruplex (termed D1-G4), (2) a weak signal induced by the single-stranded structure (termed D1-ss) and (3) almost no signal induced by the $D 1 D 2$ complex. Therefore, a ternary logic unit can be constructed based on this platform, and the outputs can be defined as "2", "1", and " 0 ", according to the fluorescence strength.

As shown in Fig. 2e, MTC is defined as the initial state. With respect to the input $X, D 1$ is defined as " 1 " and further addition of $\mathrm{Pb}^{2+}$ as "2". With respect to the input $Y$, the mixture of $D 2$ and $\mathrm{Ag}^{+}$is defined as "1" and $D 2$ alone as "2". In this system, there are five DNA motifs, including D1-ss, D1-G4 induced by $\mathrm{Pb}^{2+}$, single-stranded $D 2$ (termed D2-ss), i-motif $D 2$ induced by $\mathrm{Ag}^{+}$(termed $D 2-i)$ and the $D 1 D 2$ complex, and their stabilities are in the following order: D2-i $>D 1 D 2>D 1-G 4>D 1$-ss/D2-ss. The confirmation of each DNA structure and the thermal stability experiment results are shown in Supplementary Figs. S10-S13.

As shown in Fig. $2 \mathrm{f}$ and Supplementary Fig. S12, in the case of input $X=$ "0", the system contains no DNA, D2-i or $D 2$-ss with different input $Y$ situations. Since neither $D 2-i$ nor D2-ss can induce MTC to generate a fluorescence signal, all the outputs are " 0 ". In the case of input $X=$ "1", owing to the absence of $\mathrm{Pb}^{2+}, D 1$-ss causes a weak signal (output $=$ " 1 ") in the $(1,0)$ input situation. For the input of $(1,2), D 1$ is totally complemented by $D 2$, and the D1D2 complex decreases the output signal to "0". For the input of $(1,1)$, since $D 2-i$ is more stable than $D 1 D 2$, part of $D 1 D 2$ is unwound by $\mathrm{Ag}^{+}$, and the released D1-ss causes a weak signal, which is similar to the $(1,0)$ input situation (output $=$ " 1 "). In the case of input channel $X=$ "2", the D1-G4 induced by $\mathrm{Pb}^{2+}$ causes a strong signal (output $=" 2$ ") in the $(2,0)$ input situation. For the input $(2,2)$, since $D 1 D 2$ is more stable than D1-G4,D1-G4 is totally complemented by $D 2$, which leads to no signal (output $=$ "0"). For the input $(2,1)$, which is similar to the case of $(1,1)$, partial $D 1$ is released and forms the G-quadruplex in the presence of $\mathrm{Pb}^{2+}$. Since only some $D 1-G 4$ is formed, the signal of $M T C$ is still weak compared with that observed in the case of $(2,0)$, and the output is " 1 ". The normalized MTC FI values are plotted as a bar chart in Fig. $2 \mathrm{~g}$, and the results are accordance with the proper execution of a ternary INHIBIT gate.

\section{Data selection: the multiplexer and demultiplexer}

The multiplexer (MUX) and demultiplexer (DEMUX) are controlled rotary switches and play key roles in electronic, telecommunication, and signal processing systems $^{35}$. They involve a couple of data input/output channels and an additional address (A) control. Depending on the state of $\mathrm{A}$, data can be transmitted between certain input and output channels. To date, several MUX/ DEMUX systems have been designed based on organic molecules, enzyme reactions, or DNA platforms ${ }^{8,35-37}$. Among them, an oligonucleotide-triggered DNA conformational change is a common strategy. However, the interference among DNA strands increases the difficulty of the implementation process and limits the operational performance. In this work, owing to the multiple assembly states of MTC, a series of MUX and DEMUX was constructed in a simple manner.

The simplest MUX contains two data inputs, one output channel, and an A control (termed 2-to-1 MUX). The function of the 2-to-1 MUX is selecting one certain of the two data inputs according to the "state" of A (Fig. 3d). Figure 3a presents the schematic illustration of the constructed 2-to-1 MUX. The mixture of MTC and D1 is considered the initial state, and $D 2$ is the A control. To balance the output signal, the $D 2$ sample contains $30 \mathrm{mM}$ $\mathrm{KCl}$, which can facilitate the formation of D1-G4. Two ions, i.e., $\mathrm{Pb}^{2+}$, which can induce $D 1$ to fold into the Gquadruplex, and $\mathrm{H}^{+}$, which can induce $D 2$ to fold into the i-motif, are chosen as the two data input stimuli. In the case of $A=$ " 0 ", inputting $\mathrm{Pb}^{2+}$ promotes the formation of D1-G4 and turns on the fluorescence of MTC, while inputting $\mathrm{H}^{+}$cannot change the structure of $D 1$ nor the MTC fluorescence (Fig. 3b). In this way, the input of $\mathrm{Pb}^{2+}$ is transmitted into the MTC fluorescence signal, and the $\mathrm{H}^{+}$channel has no influence on the output. In the case of $A=$ "1", the presence of $D 2$ induces the formation of the $D 1 D 2$ complex, which can be unwound by $\mathrm{H}^{+}$but not $\mathrm{Pb}^{2}$ ${ }^{+}$. Therefore, only the acidic condition can release $D 1$. The D1-G4 induced by $\mathrm{KCl}$ can turn on the MTC 

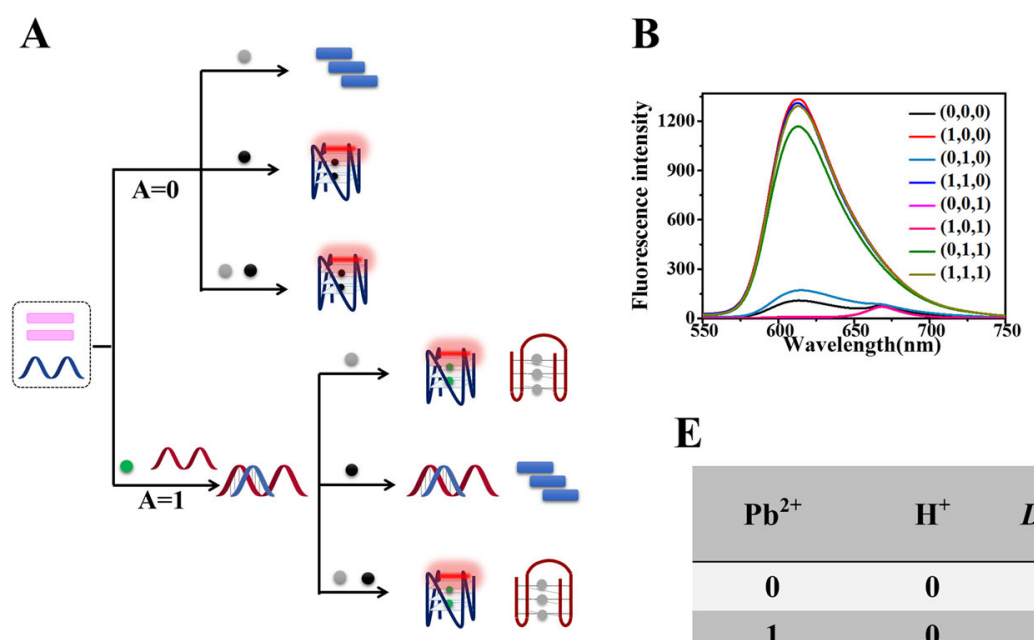

\section{C}

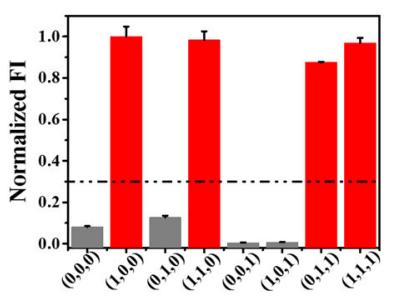

$\mathbf{E}$

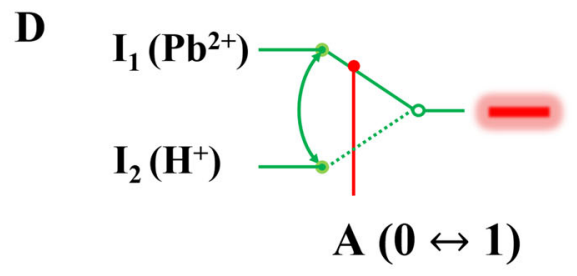

\begin{tabular}{ccc|c}
$\mathbf{P b}^{2+}$ & $\mathbf{H}^{+}$ & $D 2+K^{+}(\mathbf{A})$ & $\begin{array}{c}\mathbf{F I} \\
(613 \mathbf{~ n m})\end{array}$ \\
\hline $\mathbf{0}$ & $\mathbf{0}$ & $\mathbf{0}$ & $\mathbf{0}$ \\
1 & 0 & 0 & 1 \\
$\mathbf{0}$ & 1 & 0 & $\mathbf{0}$ \\
1 & 1 & 0 & 1 \\
0 & 0 & 1 & 0 \\
1 & 0 & 1 & 0 \\
0 & 1 & 1 & 1 \\
1 & 1 & 1 & 1 \\
\hline
\end{tabular}

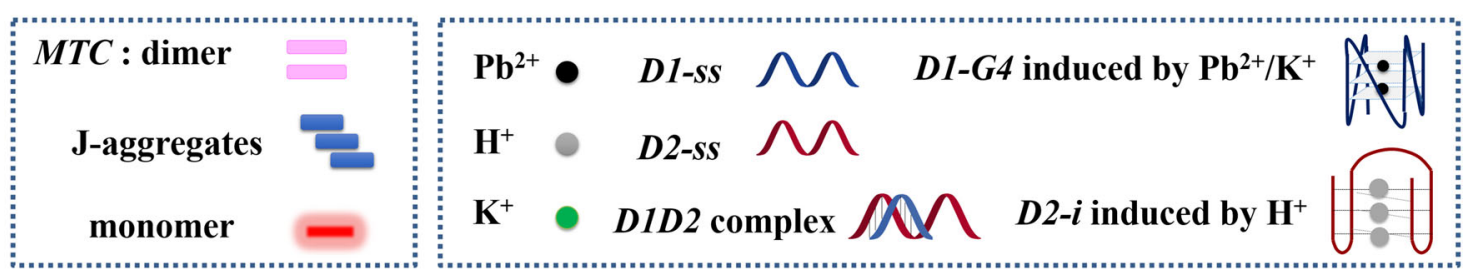

Fig. 3 Schematic illustration of the 2-to-1 MUX. a Schematic illustration of the implementation of the 2-to-1 MUX. $\mathbf{b}$ The fluorescence spectra of MTC with different input conditions. c The normalized MTC FI as the output of the 2-to-1 MUX. $\mathbf{d}$ The equivalent switching device of the 2-to-1 MUX. e The truth table of the 2-to-1 MUX logic operation

fluorescence, while the $\mathrm{Pb}^{2+}$ channel cannot influence the output. The conformational changes in MUX were confirmed by circular dichroism (CD) and native polyacrylamide gel electrophoresis (PAGE) experiments (Supplementary Figs. S14 and S15). The normalized MTC FI values are plotted as a bar chart in Fig. 3c, which satisfy the logic requirement of the 2-to-1 MUX. (The truth table is shown in Fig. 3e).

Any ion pairs capable of inducing the corresponding structural changes of $D 1 / D 2$ can be considered as inputs the 2-to-1 MUX. To demonstrate the adaptability of the platform, we also constructed another 2-to-1 MUX by changing $\mathrm{H}^{+}$to $\mathrm{Ag}^{+}$. The result is shown in Supplementary Fig. S16, and it demonstrates that the platform can be applied for parallel detection of heavy-metal $\mathrm{Pb}^{2+}$ and $\mathrm{Ag}^{+}$.

In contrast to MUX, the function of DEMUX is detangling data streams from a compressed signal. In this work, a 1-to-3 demultiplexer was also fabricated in a similar strategy (Fig. 4).

Potassium can induce MTC to form several aggregations by increasing its effective dielectric constant ${ }^{38}$, for example, $60 \mathrm{mM} \mathrm{KCl}$ can induce $M T C$ to form $\mathrm{H}$ aggregates (Supplementary Fig. S7). Herein, $\mathrm{KCl}$ is chosen as the data input and the $\mathrm{H}$-band is considered the first output channel. CE, which can chelate $\mathrm{K}^{+}$and reduce its active concentration, is chosen as one address control $\left(\mathrm{A}_{1}\right)$. When $A_{1}=$ " 1 ", inputting $\mathrm{KCl}$ leads to the formation of MTC J-aggregates. The featured J-band is considered the second output channel. In the same way, D1, which can be transformed into the G-quadruplex by $\mathrm{KCl}$ and disassemble $M T C$ aggregates into monomers, is considered another kind of address control $\left(A_{2}\right)$, and MTC FI is defined as the third output channel. The absorption and fluorescence spectra are shown in Fig. 4c, d, respectively. The normalized outputs are plotted as bar charts (Fig. 4e), 


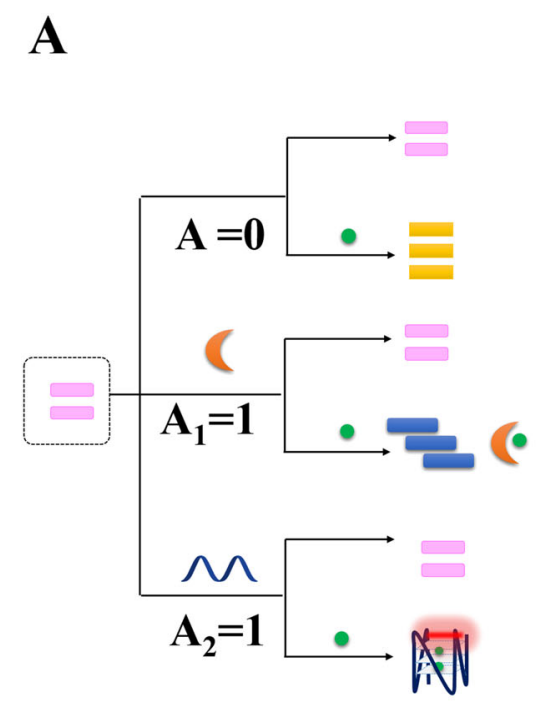

B
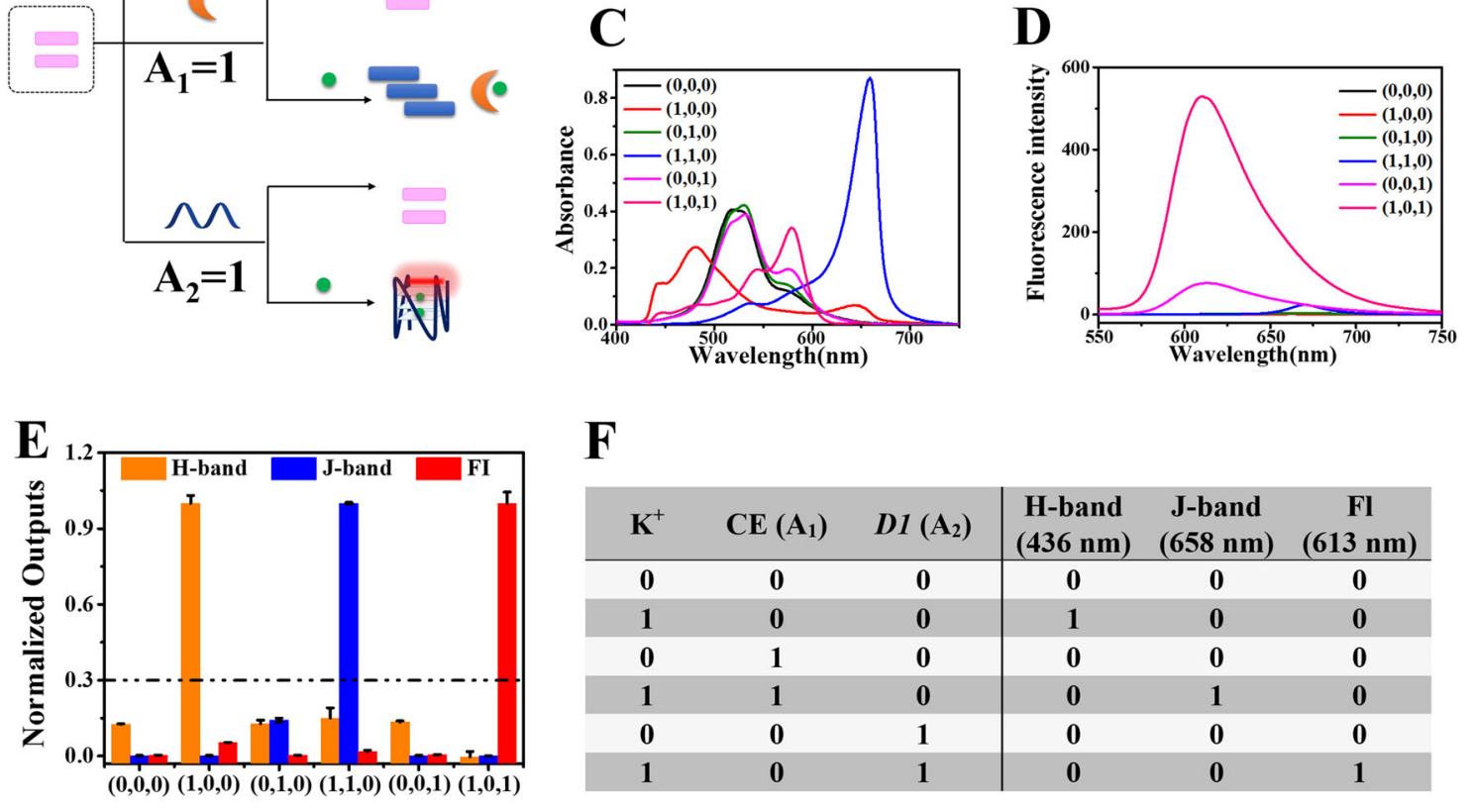

$\mathbf{F}$

\begin{tabular}{ccc|ccc}
$\mathbf{K}^{+}$ & $\mathbf{C E}\left(\mathbf{A}_{1}\right)$ & D1 $\left(\mathbf{A}_{2}\right)$ & $\begin{array}{c}\text { H-band } \\
(\mathbf{4 3 6} \mathbf{n m})\end{array}$ & $\begin{array}{c}\text { J-band } \\
(\mathbf{6 5 8} \mathbf{n m})\end{array}$ & $\begin{array}{c}\text { Fl } \\
(\mathbf{6 1 3} \mathbf{~ n m})\end{array}$ \\
\hline $\mathbf{0}$ & $\mathbf{0}$ & $\mathbf{0}$ & $\mathbf{0}$ & $\mathbf{0}$ & $\mathbf{0}$ \\
$\mathbf{1}$ & $\mathbf{0}$ & $\mathbf{0}$ & $\mathbf{1}$ & $\mathbf{0}$ & $\mathbf{0}$ \\
$\mathbf{0}$ & $\mathbf{1}$ & $\mathbf{0}$ & $\mathbf{0}$ & $\mathbf{0}$ & $\mathbf{0}$ \\
$\mathbf{1}$ & $\mathbf{1}$ & $\mathbf{0}$ & $\mathbf{0}$ & $\mathbf{1}$ & $\mathbf{0}$ \\
$\mathbf{0}$ & $\mathbf{0}$ & $\mathbf{1}$ & $\mathbf{0}$ & $\mathbf{0}$ & $\mathbf{0}$ \\
$\mathbf{1}$ & $\mathbf{0}$ & $\mathbf{1}$ & $\mathbf{0}$ & $\mathbf{0}$ & $\mathbf{1}$
\end{tabular}

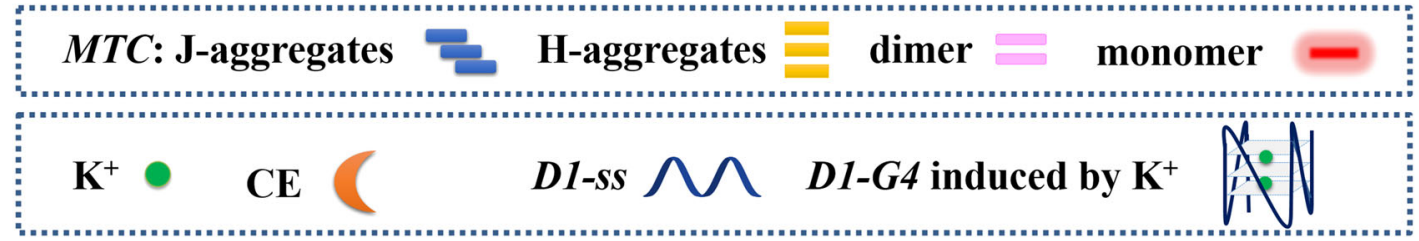

Fig. 4 Schematic illustration of the 1-to-3 DEMUX. a Schematic illustration of the implementation of the 1-to-3 DEMUX. $\mathbf{b}$ The equivalent switching device of the 1-to-3 DEMUX. The absorption (c) and fluorescence (d) spectra of MTC with different input conditions. e The normalized MTC J-band, $\mathrm{H}$-band, and Fl as the outputs of the 1-to-3 DEMUX. $\mathbf{f}$ The truth table of the 1-to-3 DEMUX logic operation

and the obtained truth table (Fig. 4f) presents the input data leads to three different output channels according to the A conditions.

\section{Data verification: the parity checker and comparator}

In data transmission, an inevitable problem is incorrect bits $^{39}$, which have serious effects on the stable performance of logic computing, especially in sophisticated circuits. A parity checker is a logic unit that can determine erroneous bits and maintain data validity. To develop a parity checker on this platform, we exploited the logic devices mentioned above with slight modifications to distinguish odd and even numbers from natural numbers between 0 and 9 (Fig. 5). The decimal numbers are encoded into four-bit binary numbers $\left(\mathrm{N}_{1}, \mathrm{~N}_{2}, \mathrm{~N}_{3}\right.$, and $\mathrm{N}_{4}$ ), and $\mathrm{H}^{+}, \mathrm{K}^{+}, \mathrm{Pb}^{2+}$, and $D 1$ are assigned to the corresponding bits for computation (Fig. 5e). Only the input situations containing $D 1$ can produce the $M T C$ fluorescence signal (Fig. 5b, c). Therefore, the corresponding fluorescence signals of all the odd numbers are extremely low, i.e., false outputs, while the even numbers produce dramatic fluorescence signals, i.e., true outputs. It is worth mentioning that the parity of a natural number, regardless of odd or even, is determined by the digit in the unit position. Thus, the logic system fabricated here can distinguish all the even numbers from the odd ones.

The comparator is another important device in signal processing systems, and it is widely used in 


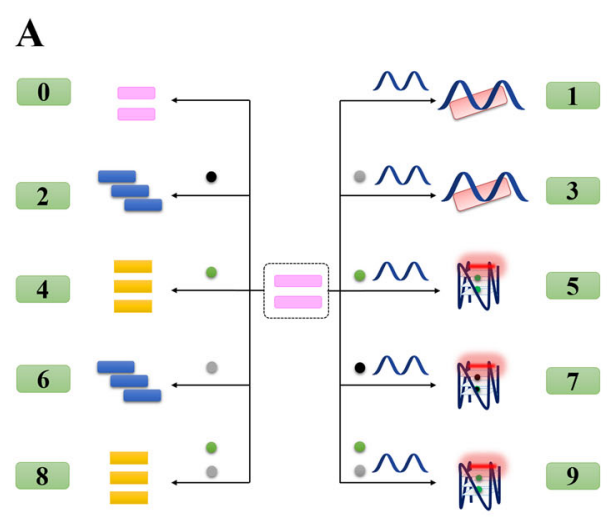

B

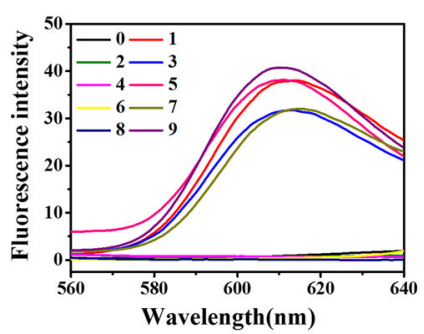

C

$\mathbf{E}$

\begin{tabular}{|c|c|c|c|c|c|}
\hline \multirow[b]{2}{*}{ Number } & \multicolumn{4}{|c|}{ Binary-coded decimal number } & \multirow{2}{*}{$\begin{array}{c}\text { Result } \\
\text { FI } \\
(613 \mathrm{~nm})\end{array}$} \\
\hline & $\begin{array}{c}\mathbf{N}_{1} \\
\left(\mathbf{H}^{+}\right)\end{array}$ & $\begin{array}{c}\mathbf{N}_{2} \\
\left(\mathbf{K}^{+}\right)\end{array}$ & $\begin{array}{c}\mathrm{N}_{3} \\
\left(\mathrm{~Pb}^{2+}\right)\end{array}$ & $\begin{array}{c}\mathbf{N}_{4} \\
(D 1)\end{array}$ & \\
\hline $\mathbf{0}$ & o & 0 & 0 & 0 & o \\
\hline 1 & $\mathbf{0}$ & $\mathbf{0}$ & 0 & 1 & 1 \\
\hline 2 & $\mathbf{0}$ & $\mathbf{0}$ & 1 & $\mathbf{0}$ & $\mathbf{0}$ \\
\hline 3 & $\mathbf{0}$ & 0 & 1 & 1 & 1 \\
\hline 4 & $\mathbf{0}$ & 1 & $\mathbf{0}$ & $\mathbf{0}$ & $\mathbf{0}$ \\
\hline 5 & $\mathbf{0}$ & 1 & $\mathbf{0}$ & 1 & 1 \\
\hline 6 & 1 & $\mathbf{0}$ & 0 & $\mathbf{0}$ & $\mathbf{0}$ \\
\hline 7 & 1 & 0 & 0 & 1 & 1 \\
\hline 8 & 1 & 1 & $\mathbf{0}$ & $\mathbf{0}$ & $\mathbf{0}$ \\
\hline 9 & 1 & 1 & 0 & 1 & 1 \\
\hline
\end{tabular}

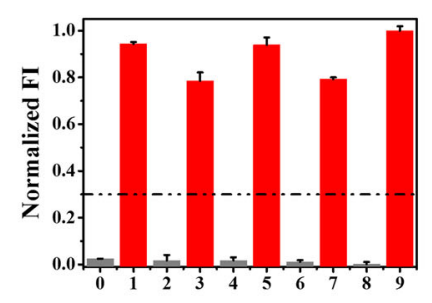

\section{$M T C:$ J-aggregates \\ $\mathbf{P b}^{2+}$ \\ $\mathbf{H}^{+} \bullet \mathbf{K}^{+}$ \\ H-aggregates \\ dimer \\ monomer \\ D1-ss ר D1-G4 induced by $\mathrm{Pb}^{2+} / \mathrm{K}^{+}$

D

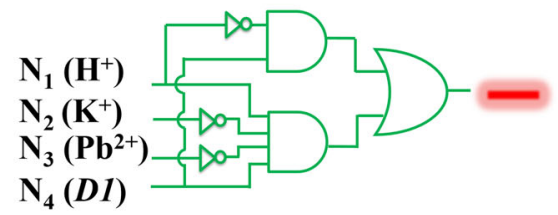

Fig. 5 Schematic illustration of the parity checker. a Schematic illustration of the implementation of the parity checker. $\mathbf{b}$ The fluorescence spectra of MTC with different input conditions. c The normalized MTC FI as the output of the parity checker. $\mathbf{d}$ Diagram of electronic parity checker logic circuit. e The truth table of the parity checker

telecommunication interfaces, analog-digital converters and sensory circuits. In this work, $\mathrm{K}^{+}$and $\mathrm{H}^{+}$are chosen as the two numbers for comparison ( $\mathrm{A}$ and $\mathrm{B}$ ), and a digital comparator is constructed based on the structural change of $D 1 D 2$ and corresponding assembly state of $M T C$ (Fig. 6).

Under our experimental conditions, $\mathrm{KCl}$ at certain concentrations can induce $M T C$ to form a mixture of $\mathrm{H}$ - and J-aggregates, while $\mathrm{H}^{+}$can induce $M T C$ to form Jaggregates. The two assembly states of MTC (J- and Haggregates) are employed as the two output channels $\left(\mathrm{O}_{2}\right.$ and $\left.\mathrm{O}_{1}\right)$, respectively. The former indicates whether $A \neq B$, and the latter indicates whether $A>B$. There are two input situations, i.e., $(0,0)$ and $(1,1)$, that lead to the result $A=B$. For the $(0,0)$ situation, the $D 1 D 2$ complex shows little interaction with $M T C$ and $M T C$ remains in the dimer form. For the $(1,1)$ situation, $\mathrm{H}^{+}$unwinds D1D2, and the released $D 1$ is induced into the G-quadruplex by $\mathrm{K}^{+}$. Consequently, $M T C$ is disassembled into monomers by D1-G4. In both situations, J- or $\mathrm{H}$-aggregates do not form, indicating that both outputs are " 0 ". In the case where A and $\mathrm{B}$ are not equal, there is no formation of D1-G4, and the assembly states of $M T C$ are determined by the cations in the system. If $A>B$, there is only $\mathrm{K}^{+}$in the system, and $M T C$ exists as a mixture of J- and $\mathrm{H}$-aggregates, thus, both outputs are " 1 ". If $A<B$, there is only $\mathrm{H}^{+}$in the system, and $M T C$ is induced to completely form J-aggregates, resulting in $O_{1}=1$ and $O_{2}=0$ (Fig. 6b, c). The conformational changes were confirmed by $\mathrm{CD}$ and PAGE experiments (Supplementary Figs. S14 and S15). The obtained truth table (Fig. 6e) presents the comparison results of the two digits, $\left(O_{1}=O_{2}=0\right)$ presents $A=B,\left(O_{1}=O_{2}=1\right)$ presents $A>B$ and $\left(O_{1}=1, O_{2}=0\right)$ presents $A<B$.

\section{Data accumulation: the counter}

In addition to combinatorial logic, sequential logic is another primary class of logic devices widely used in modern computers ${ }^{40}$. The counter is a fundamental sequential logic circuit, which is used to count stimuli pulses, frequency division, timing, and arithmetic (or logical) operations. To construct a counter on the molecular level, a logic system must fulfill the following 


\section{A}

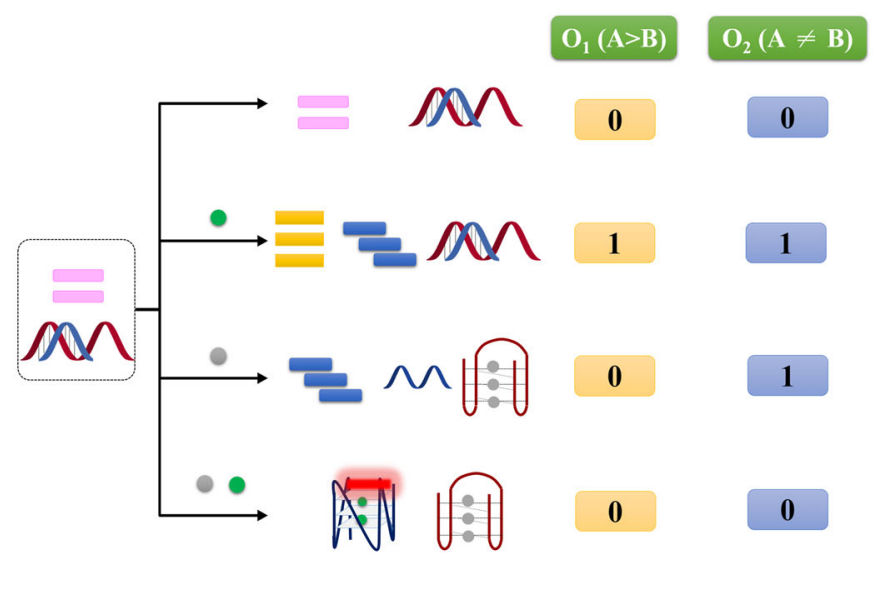

B

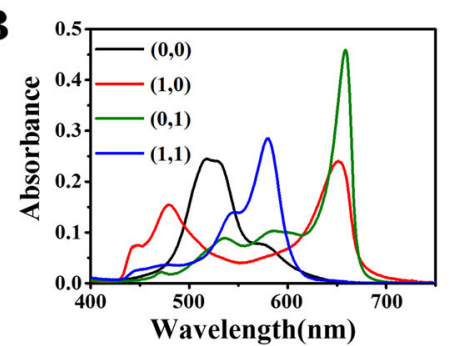

$\mathrm{C}$

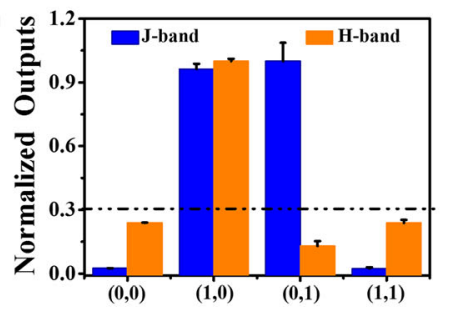

D

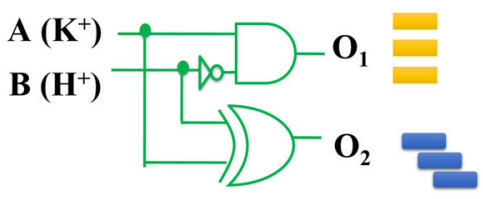

$\mathbf{E}$

\begin{tabular}{|c|c|c|c|c|}
\hline \multicolumn{3}{|c|}{ Inputs } & \multirow{2}{*}{$\begin{array}{c}\mathrm{O}_{1}(\mathrm{~A}>\mathrm{B}) \\
\text { H-band } \\
(476 \mathrm{~nm})\end{array}$} & \multirow{2}{*}{$\begin{array}{c}\mathrm{O}_{2}(\mathrm{~A} \neq \mathrm{B}) \\
\text { J-band } \\
(643 \mathrm{~nm})\end{array}$} \\
\hline Comparator & $\mathbf{A}\left(\mathrm{K}^{+}\right)$ & B $\left(\mathbf{H}^{+}\right)$ & & \\
\hline $\mathbf{A}=\mathbf{B}$ & 0 & 0 & 0 & 0 \\
\hline$A>B$ & 1 & 0 & 1 & 1 \\
\hline $\mathbf{A}<\mathbf{B}$ & 0 & 1 & 0 & 1 \\
\hline$A=B$ & 1 & 1 & 0 & 0 \\
\hline
\end{tabular}

Fig. 6 Schematic illustration of the comparator. a Schematic illustration of the implementation of the comparator. $\mathbf{b}$ The absorption spectra of MTC with different input conditions. $\mathbf{c}$ The normalized MTC J-band and H-band as the outputs of the comparator. $\mathbf{d}$ Diagram of the electronic comparator logic circuit. e The truth table of the comparator logic operation

requirements: (1) multi-channel compatibility; (2) transformations among different output channels triggered by the same stimulus at different amounts; (3) availability of chemical or biochemical pairs with opposite reactions to the system. These requirements are so challenging to simultaneously satisfy that there are few works on the construction of a molecular counter. Here, on the MTC platform, we designed a two-bit counter that can perform both cumulating and descending operations (Fig. 7).

As shown in Fig. 7e, the assembly states of $M T C$, i.e., Jand $\mathrm{H}$-aggregates, are assigned as the two bits of counted number (R), while $\mathrm{K}^{+}$and $\mathrm{CE}$ are chosen to represent the cumulating and descending operations, respectively. For the accumulating operation, inputting $\mathrm{KCl}$ pulses would induce $M T C$ to form J-aggregates alone, a mixture of Jand $\mathrm{H}$-aggregates or $\mathrm{H}$-aggregates alone step by step, indicating that the values of $R$ cumulate from 0 to 3 , respectively (Fig. 7c). With respect to the descending operation, $\mathrm{CE}$ is gradually inputted to chelate $\mathrm{K}^{+}$, and the assembly states of $M T C$ are induced backward, i.e., from $\mathrm{H}$-aggregates alone to a mixture of $\mathrm{J}$ - and $\mathrm{H}$-aggregates, $\mathrm{J}$ aggregates alone, and finally to dimers, indicating that the values of $R$ descend from 3 to 0 , respectively (Fig. 7f). The normalized MTC J- and H-bands are plotted as a bar chart in Fig. 7d, and the obtained truth table (Fig. 7e) demonstrates that the assemble states of MTC could indicate both cumulating and descending results. The results show that cumulating/descending operations can be performed at any stage of counting $R$ values in the range of $0-3$.

\section{Application perspectives}

From the viewpoint of practical applications, the developed DNA-supramolecule logic platform not only performs diverse intelligent logic functions but also shows 
A

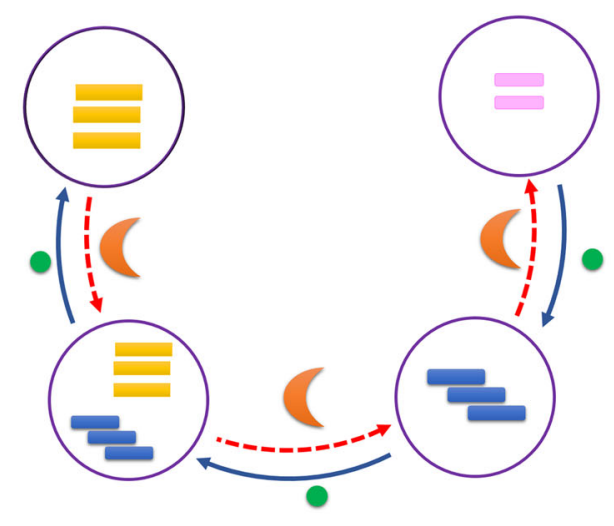

C

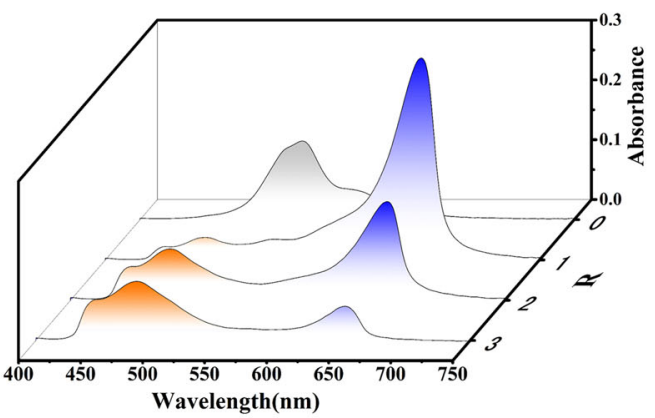

B

\section{Cumulating Descending}

$\left(\mathrm{K}^{+}\right)$

(CE)
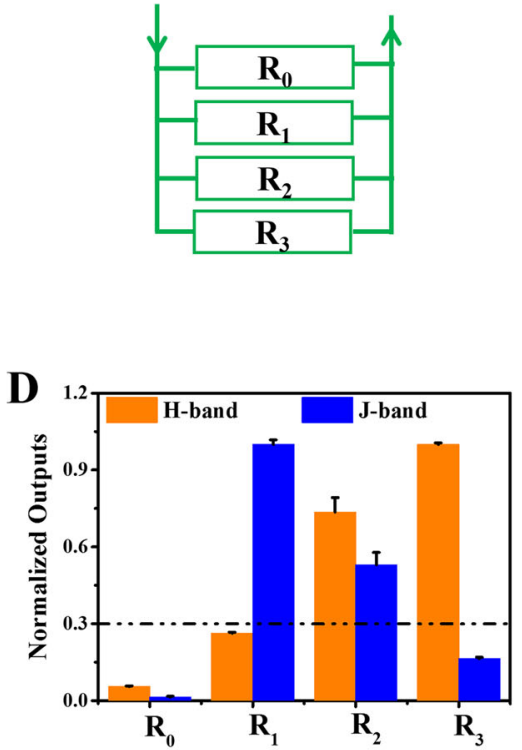

$\mathbf{E}$

\begin{tabular}{c|c|c|c|c|} 
& \multicolumn{5}{c|}{$\mathbf{K}^{+}$} \\
\hline Outputs & $\mathbf{R}_{\mathbf{0}}$ & $\mathbf{R}_{\mathbf{1}}$ & $\mathbf{R}_{\mathbf{2}}$ & $\mathbf{R}_{\mathbf{3}}$ \\
\hline J-band (654 nm) & $\mathbf{0}$ & $\mathbf{1}$ & $\mathbf{1}$ & $\mathbf{0}$ \\
\hline H-band (440 nm) & $\mathbf{0}$ & $\mathbf{0}$ & 1 & 1 \\
\hline
\end{tabular}

F

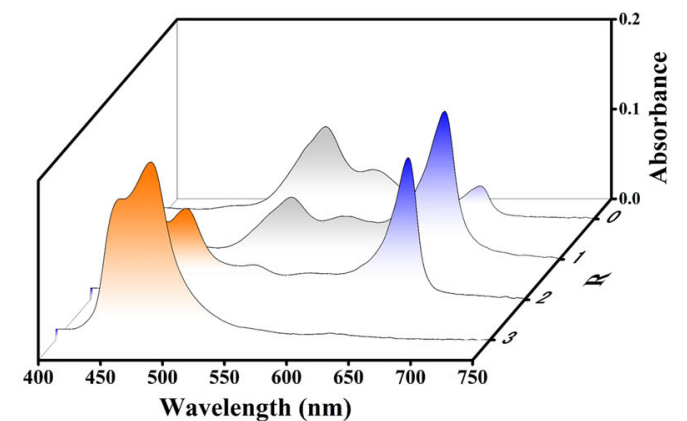

G

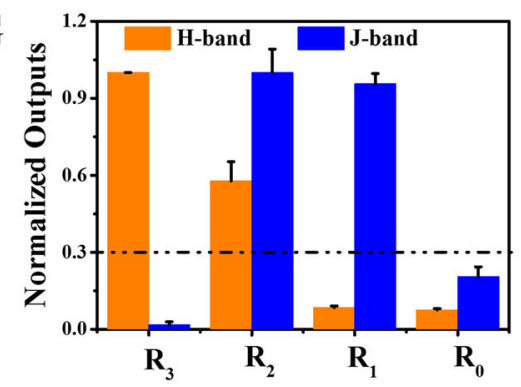

\section{$\mathrm{K}^{+} \quad \mathrm{CE}\left(\right.$ MTC: J-aggregates $ᄅ$ H-aggregates ${ }^{-}$dimer $\rightleftharpoons$ monomer}

Fig. 7 Schematic illustration of the counter. a Schematic illustration of the implementation of the counter. $\mathbf{b}$ The equivalent switching device of the counter. $\mathbf{c}$ The absorption spectra of MTC with different input conditions and (d) the normalized MTC J-band and H-band as the outputs of the cumulating process. $\mathbf{f}$ The absorption spectra of MTC with different input conditions and (g) the normalized MTC J-band and H-band as the outputs of the descending process. e The truth table of the counter logic operation 

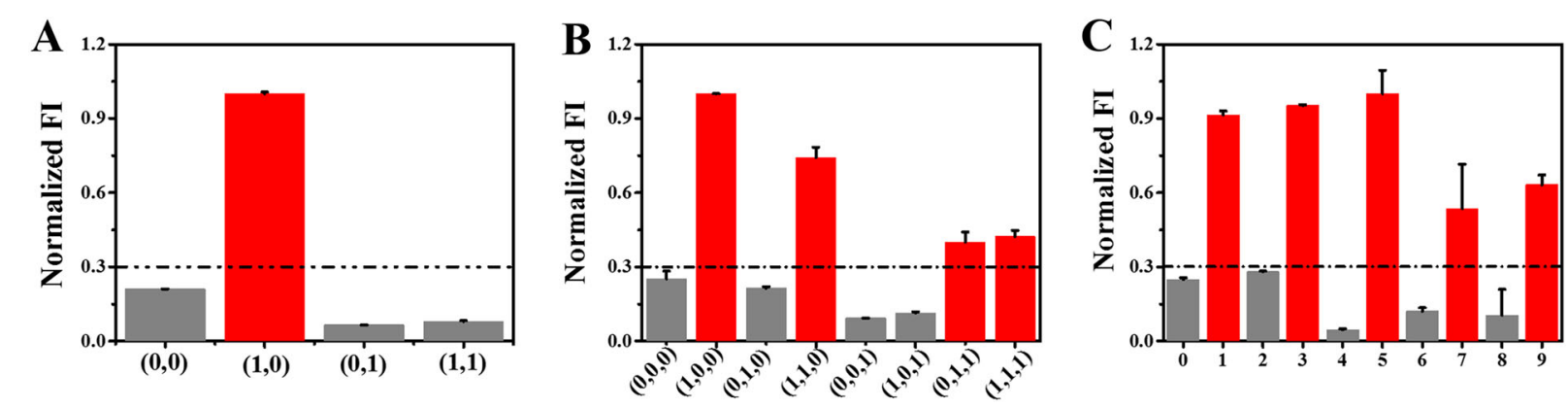

Fig. 8 The normalized fluorescence intensity (FI) of $M T C$ monomers as the output. a The binary INHIBIT, b 2-to-1 multiplexer (MUX), and $\mathbf{c}$ parity checker implemented in human serum samples diluted 1000 times with Tris-HCl buffer solution

great potential for applications in multiplex chemical analysis. For example, the binary INHIBIT gate can analyze $\mathrm{Pb}^{2+}$ with high efficiency, and the MUX can parallelly discriminate $\mathrm{Pb}^{2+}$ and $\mathrm{Ag}^{+}$. As highly poisonous metal elements, lead harms almost every organ and system in organisms, especially the nervous system ${ }^{41}$, while silver can cause severe damage to cell membranes via extracellular mechanisms, inducing skin irritation, organ edema, and stomach distress ${ }^{42,43}$. Excessive amounts of both ions are lethal. Lead, silver, and their compounds are prevalently applied in electrical, electronic, biomedical, jewelry, imaging industries, and manufacturing fungicides, and they usually coexisting in various sources like water, soil, and industrial waste. Therefore, integrated sensing methods that can parallelly detect these heavy-metal ions show much wider application prospects than single-target methods for these individual metals. To date, only a few parallel detection methods have been reported ${ }^{44-46}$. For example, Pei et al. ${ }^{46}$ designed a DNA nanostructured microarray (DNM) that could sensitively and selectively detect multiple heavy-metal ions $\left(\mathrm{Hg}^{2+}, \mathrm{Pb}^{2+}\right.$, and $\left.\mathrm{Ag}^{+}\right)$. The 2-to-1 MUX constructed in this work can be applied to analyze $\mathrm{Pb}^{2+}$ or $\mathrm{Ag}^{+}$, and the method is efficient, lowcost and convenient for on-site detection. Moreover, the two MUX circuits with different inputs indicate the flexibility and wide adaptability of this platform for multiplex chemical and biochemical analyses.

Furthermore, the development of DNA-based logic systems will be crucial for applications in complex biological environments. To expand the potential application scenarios of the MTC-DNA platform, the binary INHIBIT, 2-to-1 MUX and parity checker were also operated in diluted human serum. As shown in Fig. 8, all the results satisfy the logic requirements, and all the logic devices present the same threshold value (spectral results shown in Supplementary Fig. S17). The results suggest that the proposed logic platform shows great adaptability for complex biological environments and has potential for clinical diagnosis applications.

\section{Conclusions}

In conclusion, by combining DNA structural changes with supramolecule assembly, we developed a versatile logic platform to construct combinational and sequential logic circuits for information processing, including binary and ternary INHIBIT gates, two 2-to-1 MUX, a 1-to-3 DEMUX, a parity checker, a comparator and a counter. Since the DNA structures and supramolecule assembly states can be simultaneously regulated by the inputs, all the logic operations were implemented with high efficiency (all logic operations completed within $30 \mathrm{~min}$ ) and excellent performance (constant threshold as low as 0.3). Furthermore, the logic system not only performs various intelligent logic functions but also shows potential applications for multiplex chemical analysis and clinical diagnosis. The developed DNA-supramolecule platform provides a novel prototype for the design of multifunctional molecular logic circuits, which may bridge the gap between molecular devices and silicon-based electronics owing to its high reconfigurability, flexibility, parallelism, scalability, and considerable application prospects.

\section{Acknowledgements}

This work was supported by the National Natural Science Foundation of China (31400702) and the Fundamental Research Funds for the Central Universities. The kind assistance of Prof. Peng Wu from the Analytical \& Testing Center at Sichuan University with the CD measurements was greatly appreciated.

\footnotetext{
Author details

${ }^{1}$ College of Chemistry, Sichuan University, Chengdu 610064, China. ${ }^{2}$ Key Laboratory of Drug Targeting and Drug Delivery Systems, West China School of Pharmacy, Sichuan University, Chengdu 610041, China. ${ }^{3}$ West China School of Public Health, No.4 West Teaching Hospital, Sichuan University, Chengdu 610041, China. ${ }^{4}$ Dalian Ocean University, Dalian 116023, China. ${ }^{5}$ National Laboratory for Molecular Sciences, Centre for Molecular Sciences, State Key Laboratory for Structural Chemistry of Unstable and Stable Species, Institute of Chemistry, Chinese Academy of Sciences, Beijing 100190, China
}

Conflict of interest

The authors declare that they have no conflict of interest. 


\section{Publisher's note}

Springer Nature remains neutral with regard to jurisdictional claims in published maps and institutional affiliations.

Supplementary information is available for this paper at https://doi.org/ 10.1038/s41427-018-0051-4.

Received: 29 November 2017 Accepted: 2 April 2018. Published online: 6 June 2018

\section{References}

1. Katz, E. \& Privman, V. Enzyme-based logic systems for information processing. Chem. Soc. Rev. 39, 1835-1857 (2010).

2. Andreasson, J. \& Pischel, U. Smart molecules at work-mimicking advanced logic operations. Chem. Soc. Rev. 39, 174-188 (2010).

3. Pu, F., Ren, J. \& Qu, X. Nucleic acids and smart materials: advanced building blocks for logic systems. Adv. Mater. 26, 5742-5757 (2014).

4. Pu, F., Ju, E., Ren, J. \& Qu, X. Multiconfigurable logic gates based on fluorescence switching in adaptive coordination polymer nanoparticles. Adv. Mater. 26, 1111-1117 (2014)

5. Pu, F., Ren, J. \& Qu, X. "Plug and play" logic gates based on fluorescence switching regulated by self-assembly of nucleotide and lanthanide ions. ACS Appl. Mater. Interfaces 6, 9557-9562 (2014).

6. Orbach, R., Remacle, F., Levine, R. D. \& Willner, I. DNAzyme-based 2:1 and 4:1 multiplexers and 1:2 demultiplexer. Chem. Sci. 5, 1074 (2014).

7. Kang, D. et al. DNA biomolecular-electronic encoder and decoder devices constructed by multiplex biosensors. NPG Asia Mater. 4, e1 (2012).

8. Li, H., Liu, Y., Dong, S. \& Wang, E. DNA-based advanced logic circuits for nonarithmetic information processing. NPG Asia Mater. 7, e166 (2015).

9. Zhang, S. et al. A label-free and universal platform for the construction of an odd/even detector for decimal numbers based on graphene oxide and DNAstabilized silver nanoclusters. Nanoscale 9, 11912-11919 (2017).

10. Fan, D., Wang, E. \& Dong, S. Exploiting polydopamine nanospheres to DNA computing: A simple, enzyme-free and G-quadruplex-free DNA parity generator/checker for error detection during data transmission. ACS Appl. Mater. Interfaces 9, 1322-1330 (2017).

11. Mohandoss, S., Sivakamavalli, J., Vaseeharan, B. \& Stalin, T. Host-guest molecular recognition based fluorescence On-Off-On chemosensor for nanomolar level detection of $\mathrm{Cu}^{2+}$ and $\mathrm{Cr}_{2} \mathrm{O}_{7}{ }^{2-}$ ions: Application in XNOR logic gate and human lung cancer living cell imaging. Sens. Actuators B 234, 300-315 (2016).

12. Gaber, R. et al. Designable DNA-binding domains enable construction of logic circuits in mammalian cells. Nat. Chem. Biol. 10, 203-208 (2014).

13. You, M. et al. DNA "nano-claw": logic-based autonomous cancer targeting and therapy. J. Am. Chem. Soc. 136, 1256-1259 (2014).

14. Xu, W., Deng, R., Wang, L. \& Li, J. Multiresponsive rolling circle amplification for DNA logic gates mediated by endonuclease. Anal. Chem. 86, 7813-7818 (2014).

15. Gao, R. R., Shi, S., Zhu, Y., Huang, H. L. \& Yao, T. M. A RET-supported logic gate combinatorial library to enable modeling and implementation of intelligent logic functions. Chem. Sci. 7, 1853-1861 (2016).

16. Liu, C., Yang, D., Jin, Q., Zhang, L. \& Liu, M. A chiroptical logic circuit based on self-assembled soft materials containing amphiphilic spiropyran. Adv. Mater. 28, 1644-1649 (2016).

17. Xie, Y. J. et al. An elaborate supramolecular assembly for a smart nanodevice for ratiometric molecular recognition and logic gates. Chem. Eur. J. 22, 8339-8345 (2016).

18. Jones, R. M. et al. Building highly sensitive dye assemblies for biosensing from molecular building blocks. Proc. Natl Acad. Sci. USA 98, 14769-14772 (2001).

19. Wurthner, F., Yao, S., Debaerdemaeker, T. \& Wortmann, R. Dimerization of merocyanine dyes. Structural and energetic characterization of dipolar dye aggregates and implications for nonlinear optical materials. J. Am. Chem. Soc. 124, 9431-9447 (2002).

20. Hranisavljevic, J., Dimitrijevic, N. M., Wurtz, G. A. \& Wiederrecht, G. P. Photoinduced charge separation reactions of J-aggregates coated on silver nanoparticles. J. Am. Chem. Soc. 124, 4536-4537 (2002).

21. Mishra, A., Behera, R. K., Behera, P. K., Mishra, B. K. \& Behera, G. B. Cyanines during the 1990s: A review. Chem. Rev. 100, 1973-2011 (2000).
22. Yang, C. et al. A novel reconfigurable logic unit based on the DNA-templated potassium-concentration-dependent supramolecular assembly. Chem. Eur. J. 24, 4019-4025 (2018).

23. Sun, $\mathrm{H}$. et al. Controllable assembly and cycling conversion of various supramolecular aggregates of a cyanine dye. Appl. Phys. Lett. 98, 031103 (2011).

24. Sun, $H$. et al. A colorimetric temperature sensor of a cyanine dye supramolecule and its application in reversible switch. Appl. Phys. Lett. 105, 071914 (2014).

25. Zhang, Y., Xiang, J., Tang, Y., Xu, G. \& Yan, W. Aggregation behaviour of two thiacarbocyanine dyes in aqueous solution. Dyes Pigm. 76, 88-93 (2008).

26. Shen, G., Zhang, H., Yang, C., Yang, Q. \& Tang, Y. Thrombin ultrasensitive detection based on chiral supramolecular assembly signal-amplified strategy induced by thrombin-binding aptamer. Anal. Chem. 89, 548-551 (2017).

27. Yang, Q. et al. Verification of specific G-quadruplex structure by using a novel cyanine dye supramolecular assembly: II. The binding characterization with specific intramolecular G-quadruplex and the recognizing mechanism. Nucl. Acids Res. 38, 1022-1033 (2010).

28. Gai, W. et al. A dual-site simultaneous binding mode in the interaction between parallel-stranded G-quadruplex [d(TGGGGT)]4 and cyanine dye 2,2'diethyl-9-methyl-selenacarbocyanine bromide. Nucl. Acids Res. 41, 2709-2722 (2013).

29. Hamer, F. M. The Cyanine Dyes and Related Compounds. (Interscience Publishers, New York, 1964).

30. Ficken, G. E. The Chemistry of Synthetic Dyes. (Academic Press, New York, 1971).

31. Wang, L. X. et al. Controllable cy3-MTC-dye aggregates and its applications served as a chemosensor. Dyes Pigm. 122, 382-388 (2015).

32. Yang, Q. F. et al. Verification of intramolecular hybrid/parallel G-quadruplex structure under physiological conditions using novel cyanine dye $\mathrm{H}$-aggregates: Both in solution and on Au film. Anal. Chem. 82, 9135-9137 (2010).

33. Sun, $\mathrm{H}$. et al. Quantification of the $\mathrm{Na}^{+/} \mathrm{K}^{+}$ratio based on the different response of a newly identified G-quadruplex to $\mathrm{Na}^{+}$and $\mathrm{K}^{+}$. Chem. Commun. 49, 4510-4512 (2013).

34. Ran, X., Pu, F., Ren, J. \& Qu, X. DNA-regulated upconverting nanoparticle signal transducers for multivalued logic operation. Small 10, 1500-1503 (2014).

35. Amelia, M., Baroncini, M. \& Credi, A. A simple unimolecular multiplexer/ demultiplexer. Angew. Chem. Int. Ed. 47, 6240-6243 (2008).

36. Andreasson, J. et al. Molecular 2: 1 digital multiplexer. Angew. Chem. Int. Ed. 46, 958-961 (2007).

37. Wang, K., He, M., Wang, J., He, R. \& Wang, J. Implementation of arithmetic and nonarithmetic functions on a label-free and DNA-based platform. Sci. Rep. 6, 34810 (2016).

38. Robinson, B. H., Loffler, A. \& Schwarz, G. Thermodynamic behavior of acridineorange in solution-model system for studying stacking and charge-effects on self-aggregation. J. Chem. Soc. Farad. 69, 56-69 (1973).

39. Bhattacharyya, A., Kumar Gayen, D. \& Chattopadhyay, T. All-optical parallel parity generator circuit with the help of semiconductor optical amplifier (SOA)-assisted Sagnac switches. Opt. Commun. 313, 99-105 (2014).

40. Rout, B., Milko, P., Iron, M. A., Motiei, L. \& Margulies, D. Authorizing multiple chemical passwords by a combinatorial molecular keypad lock. J. Am. Chem. Soc. 135, 15330-15333 (2013).

41. Needleman, H. Lead poisoning. Ann. Rev. Med. 55, 209-222 (2004).

42. Wood, C. M., Hogstrand, C., Galvez, F. \& Munger, R. S. The physiology of waterborne silver toxicity in freshwater rainbow trout (Oncorhynchus mykiss) 1. The effects of ionic $\mathrm{Ag}^{+}$. Aquat. Toxicol. 35, 93-109 (1996).

43. Kumar, M., Kumar, R. \& Bhalla, V. Optical Chemosensor for $\mathrm{Ag}+$, Fe3 +, and Cysteine: Information Processing at Molecular Level. Org. Lett. 13, 366-369 (2011).

44. Wang, S.-e. \& Si, S. Aptamer biosensing platform based on carbon nanotube long-range energy transfer for sensitive, selective and multicolor fluorescent heavy metal ion analysis. Anal. Methods 5, 2947-2953 (2013).

45. Xia, J. et al. Metal ion-mediated assembly of DNA nanostructures for cascade fluorescence resonance energy transfer-based fingerprint analysis. Anal. Chem. 86, 7084-7087 (2014).

46. Qu, X. et al. Bubble-mediated ultrasensitive multiplex detection of metal ions in three-dimensional DNA nanostructure-encoded microchannels. ACS Appl. Mater. Interfaces 9, 16026-16034 (2017). 